\title{
BMJ Open Association between facility-level adherence to phosphorus management guidelines and mortality in haemodialysis patients: a prospective cohort study
}

\author{
Takahiro Itaya (D) , ' Sayaka Shimizu (D) , ,3 Takashi Hara, ${ }^{1}$ Yoshinori Matsuoka, ${ }^{1,4}$ \\ Shunichi Fukuhara, ${ }^{2,5,6}$ Yosuke Yamamoto (D) ${ }^{1}$
}

To cite: Itaya T, Shimizu S, Hara T, et al. Association between facility-level adherence to phosphorus management guidelines and mortality in haemodialysis patients: a prospective cohort study. BMJ Open 2021;11:e051002. doi:10.1136/ bmjopen-2021-051002

- Prepublication history and additional supplemental material for this paper are available online. To view these files, please visit the journal online (http://dx.doi.org/10.1136/ bmjopen-2021-051002).

Received 06 March 2021 Accepted 19 August 2021

D) Check for updates

(c) Author(s) (or their employer(s)) 2021. Re-use permitted under CC BY-NC. No commercial re-use. See rights and permissions. Published by BMJ.

For numbered affiliations see end of article.

\section{Correspondence to}

Dr Yosuke Yamamoto;

yamamoto.yosuke.5n@kyoto-u. ac.jp

\section{ABSTRACT}

Objectives To examine the association between facilitylevel adherence to phosphorus management guidelines and mortality among patients with haemodialysis, and to explore the facility-related factors associated with facilitylevel guideline adherence.

Design Prospective cohort study.

Setting The Dialysis Outcomes and Practice Pattern Study, which included 57 representative dialysis facilities in Japan between 2012 and 2015.

Participants A total of 2054 adult patients who received maintenance haemodialysis were included. We defined exposure according to the following four categories, depending on whether facility-level target ranges of serum phosphorus concentration adhered to the Japanese clinica practice guidelines: adherence group (lower limit $\geq 3.5 \mathrm{mg}$ / $\mathrm{dL}$ and upper limit $\leq 6.0 \mathrm{mg} / \mathrm{dL}$ ), low-target group (lower limit $<3.5$ and upper limit $\leq 6.0$ ), wide-target group (lower limit $<3.5$ and upper limit $>6.0$ ) and high-target group (lower limit $\geq 3.5$ and upper limit $>6.0$ ).

Primary outcome measure The primary outcome was the patient all-cause mortality rate.

Results The mortality rate among the patients was 7.3 per 100 person-years; 27 facilities (47\%) set targets according to the guidelines. HRs for mortality with reference to the adherence group were $1.04(95 \% \mathrm{Cl} 0.76$ to 1.43) in the low-target group, $1.11(95 \% \mathrm{Cl} 0.68$ to $1.81)$ in the wide-target group and $1.95(95 \% \mathrm{Cl} 1.12$ to 3.38 ) in the high-target group. Involvement of dieticians in dialysis treatment was associated with facility-level guideline adherence (OR 4.51; 95\% $\mathrm{Cl} 1.15$ to 17.7). Conclusions A higher facility-level target range for phosphorus was associated with increased patient mortality. Among facilities that set the target according to the guidelines, dieticians tended to be involved in dialysis care. These findings suggest the importance of reviewing facilities' treatment policies in relation to updated guidelines and the need to work with relevant professionals.

\section{INTRODUCTION}

Clinical practice guidelines (CPGs) play an important role in reducing inappropriate

\section{Strengths and limitations of this study}

- A major strength of this study is that we analysed a large sample of representative dialysis facilities in Japan; therefore, we could examine facility-level factors.

- The measurement of exposure and facility-level treatment policy had high validity, as the data were collected prospectively to prevent recall bias.

- The facility-level treatment policy reported by facility directors might not represent an aggregate opinion in each facility.

variations in clinical practice, thereby improving the quality of care and decreasing healthcare expenditures. ${ }^{1-6}$ In recent years, many clinical societies have shown high interest in CPGs, and have developed and published CPGs in various clinical areas. CPGs are widely used in quality management and healthcare policy. However, adherence to CPGs differs considerably, with several studies reporting that adherence percentages varied from $10 \%$ to $80 \% .^{78}$ The impact of CPGs is dependent on their acceptance by clinicians in daily practice. Accordingly, research into whether clinicians practice according to the guidelines has received scientific and political interest. $^{9}$

Dialysis treatment is one of the representative clinical areas for which CPGs have been generated worldwide. ${ }^{1011}$ There are many treatment goals in this area, among which the treatment of chronic kidney disease-mineral and bone disorder (CKD-MBD) has received considerable attention from nephrologists. In particular, serum phosphorus control is a major target that has been associated with prognosis. $^{1213}$ 


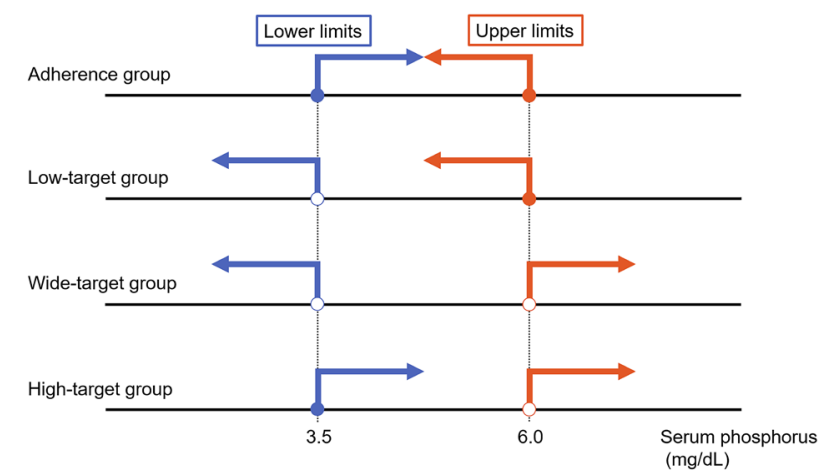

Figure 1 Definition of the exposure. Adherence group, lower limit of serum phosphorus value (LL) $\geq 3.5 \mathrm{mg} / \mathrm{dL}$ and upper limit of serum phosphorus value (UL) $\leq 6.0 \mathrm{mg} / \mathrm{dL}$; low-target group, $<3.5 \mathrm{mg} / \mathrm{dL}$ and UL $\leq 6.0 \mathrm{mg} / \mathrm{dL}$; widetarget group, $<3.5 \mathrm{mg} / \mathrm{dL}$ and $\mathrm{UL}>6.0 \mathrm{mg} / \mathrm{dL}$; high-target group, $\geq 3.5 \mathrm{mg} / \mathrm{dL}$ and UL $>6.0 \mathrm{mg} / \mathrm{dL}$. LL, lower limits; UL, upper limits.

Although almost the same treatment targets for phosphorus have been set in CPGs for phosphorus management worldwide, it is unknown whether institutional treatment policies that do not adhere to the CKD-MBD guidelines affect patient outcomes. Also, the recommendations in CPGs are not absolute, and the CPGs take into account that treatment policies will vary depending on the condition of individual patients. However, it is unclear whether differences between recommendations in CPGs and facility-level treatment policies affect patient outcomes.

The treatment policy for phosphorus management at each facility may be an appropriate indicator for assessing guideline adherence, as it can synthetically reflect CPG accessibility, attitudes of healthcare professionals and usability of medical resources. ${ }^{7} 9$ 14-21 ${ }^{\text {Furthermore, }}$ the facility characteristics associated with phosphorus management policies have not been identified.

We, therefore, examined the association between guideline adherence regarding the facility-level target range of serum phosphorus and mortality in dialysis patients, and explored the facility-related factors associated with facility-level guideline adherence.

\section{METHODS}

\section{Study design and participants}

This was a cohort study using data from the Dialysis Outcomes and Practice Pattern Study (DOPPS) in Japan. The DOPPS was a prospective cohort study of patients enrolled randomly from a representative sample of dialysis facilities within each participating country. All participants in the DOPPS provided written informed consent prior to study enrolment. We conducted this study in compliance with the Declaration of Helsinki. This study was approved by Kyoto University Graduate School and Faculty of Medicine Kyoto University Hospital
Ethics Committee. Detailed information on the design of DOPPS has been provided elsewhere. ${ }^{22} 23$

Our cohort study used data from the Japanese DOPPS (J-DOPPS) phase V conducted from 1 June 2012 to 31 May 2015. We included patients receiving maintenance haemodialysis (HD) who were older than 18 years. We excluded patients who had been receiving dialysis therapy for less than 90 days because mineral metabolism markers fluctuate more dramatically near the beginning of dialysis therapy and may not represent patterns of steady-state bone mineral dynamics. Additionally, patients with any missing data were excluded.

\section{Definition of exposure}

The exposure was the facility-level target range of serum phosphorus concentration set by each facility where patients were receiving dialysis. Facility directors reported lower limits (LL) and upper limits (UL) of serum phosphorus targets at baseline and then every 4 months during the study period. We defined the exposure according to the following four categories (figure 1), depending on whether the baseline LL and UL ranges adhered to the CPG for the management of CKD-MBD published by the Japanese Society for Dialysis Therapy in April 2012. ${ }^{24}{ }^{25}$ This CPG recommended a serum phosphorus target range from 3.5 to $6.0 \mathrm{mg} / \mathrm{dL}$.

- Adherence group: both LL and UL adhered to the values in the CPG (LL: $\geq 3.5 \mathrm{mg} / \mathrm{dL}, \mathrm{UL}: \leq 6.0 \mathrm{mg} / \mathrm{dL}$ ).

- Low-target group: LL fell below and UL adhered the values in the CPG (LL: $<3.5 \mathrm{mg} / \mathrm{dL}, \mathrm{UL}: \leq 6.0 \mathrm{mg} / \mathrm{dL}$ ).

- Wide-target group: LL fell below and UL exceeded the values in the CPG (LL: $<3.5 \mathrm{mg} / \mathrm{dL}, \mathrm{UL}:>6.0 \mathrm{mg}$ / dL).

- High-target group: LL adhered to and UL exceeded the values in the CPG (LL: $\geq 3.5 \mathrm{mg} / \mathrm{dL}, \mathrm{UL}:>6.0 \mathrm{mg} /$ dL).

\section{Outcomes}

The primary outcome was the all-cause mortality rate, indicated by HRs. The secondary outcome was major adverse cardiovascular events (MACEs), including acute myocardial infarction, stroke and all-cause mortality. ${ }^{26}$ The survival period was calculated from patient enrolment to death. Survival at the end of the observation period (31 May 2015) was censored.

\section{Covariates}

Data collected at baseline consisted of age, gender, HD vintage (years), serum albumin $(\mathrm{g} / \mathrm{dL})$, serum phosphorus $(<3.5 \mathrm{mg} / \mathrm{dL}, 3.5-6.0 \mathrm{mg} / \mathrm{dL},>6.0 \mathrm{mg} / \mathrm{dL}),{ }^{24}$ corrected serum calcium $(<8.4 \mathrm{mg} / \mathrm{dL}, 8.4-10.0 \mathrm{mg} /$ $\mathrm{dL},>10.0 \mathrm{mg} / \mathrm{dL}),{ }^{24}$ intact parathyroid hormone (PTH) $(<60 \mathrm{pg} / \mathrm{mL}, 60-240 \mathrm{pg} / \mathrm{mL},>240 \mathrm{pg} / \mathrm{mL}),{ }^{24}$ history of diabetes mellitus, history of liver disease, history of hypertension, history of heart disease (coronary heart disease, coronary artery disease, angina, myocardial infarction, bypass surgery and coronary angioplasty), history of stroke or transient ischaemic attack (TIA), history of 


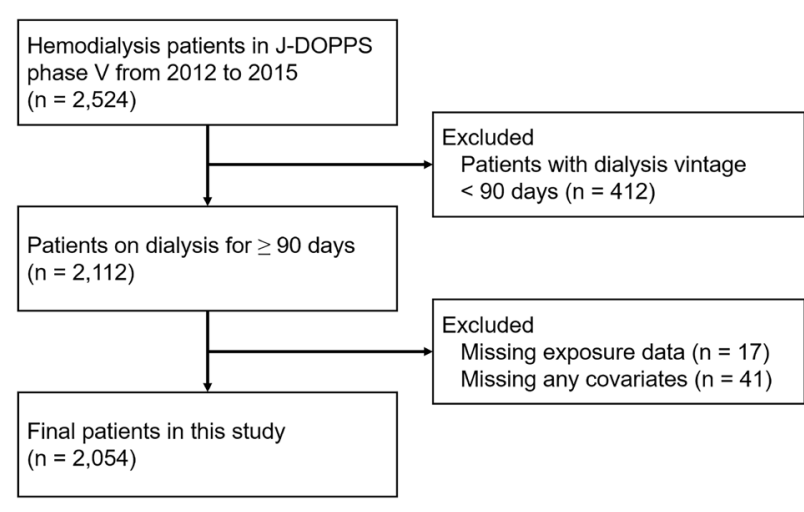

Figure 2 Selection process for study population. J-DOPPS, Japanese Dialysis Outcomes and Practice Pattern Study.

cancer, treatment time on dialysis per day $(<211 \mathrm{~min}$, 211-240 min, >240 min $)^{27}$ and CKD-MBD-related medications (phosphate binder, oral vitamin D receptor activator (VDRA), intravenous VDRA and cinacalcet). Corrected serum calcium was calculated as measured calcium concentration $(\mathrm{mg} / \mathrm{dL})+(4$-serum albumin $(\mathrm{g} /$ dL) ) when serum albumin was under $4.0 \mathrm{~g} / \mathrm{mL} .^{24} 28$

To investigate facility-level factors related to guideline adherence, we assessed facility size, organisation type (private or public), consultation time per week, presence of nocturnal dialysis programmes, and whether dietitians, nurses certified in dialysis nursing and/or certified social workers were involved in dialysis treatment. Facility size was divided into three groups according to the number of HD patients: fewer than 100 patients, $100-150$ patients and more than 150 patients. Consultation time was defined as consultation hours per week, classified as less than 40 hours, 40-60 hours and more than 60 hours per week. Nocturnal dialysis denoted that a facility offered HD treatment at night while patients slept.

\section{Statistical analysis}

The baseline characteristics were summarised for all patients and for patients categorised in the four exposure groups. Continuous variables were described as means and $\mathrm{SD}$, and categorical variables were represented as counts and percentages (\%). Differences in the distribution of patient characteristics at baseline among the four groups were assessed using one-way analysis of variance for continuous variables and the $\chi^{2}$ test for categorical variables. Also, we described the individual changes of serum phosphorus during the study period by exposures. Observation time and number of deaths and MACEs within the follow-up period were also summarised. The event-free survival rate for all-cause mortality and MACEs according to the categorised groups was described by the Kaplan-Meier method and compared using the log-rank test.

The primary analysis evaluated the association between the facility-level target range and mortality. We evaluated a mixed-effects Cox regression model with random intercept to account for potential differences among dialysis facilities. This analysis was adjusted for patientlevel covariates: age, gender, HD vintage, serum albumin, serum phosphorus, corrected serum calcium and comorbidities (diabetes mellitus, liver disease, hypertension, heart disease, stroke or TIA, and cancer). Unadjusted and adjusted HRs and 95\% CIs were estimated using the survival model. To assess the proportionality assumption of the survival model, we examined Schoenfeld residuals. The secondary analysis was used the same model and adjusted variables for investigating the association between the facility-level target range and MACEs.

The exploratory analysis examined the facility-level factors that were associated with facility-level guideline adherence. First, facility characteristics were described by frequencies and percentages. Second, we conducted univariate logistic regression analyses using a binary outcome: adherence group or not. Explanatory variables were facility size, organisation type, consultation time, availability of nocturnal dialysis, availability of dietitian and availability of certified social worker. Third, the variables that met the criterion $\mathrm{p}<0.2$ in univariate analyses were entered into the final multivariate logistic regression model. ORs and 95\% CIs were estimated and tested for statistical significance.

All statistical analyses were performed using STATA V.16.1 (StataCorp). Two-tailed p values of less than 0.05 were considered statistically significant, except for the univariate regressions of the explanatory analysis, as noted earlier.

\section{Patient and public involvement}

There was no patient and public involvement.

\section{RESULTS}

\section{Patient flow}

A total of 2524 patients participated in the study between 2012 and 2015. Patients were excluded if their history of dialysis was less than 90 days $(n=412)$ or in case of missing essential data (facility with missing exposure data: $n=17$, patients with missing covariates: $n=41$ ), resulting in 2054 patients included in our analysis (figure 2).

\section{Facility treatment policy}

In this study, $47 \%$ of facilities (27/57 facilities) adhered to the CPG for CKD-MBD; this adherence group included $941(46 \%)$ patients. There were 729 (35\%) patients in the low-target group (21 facilities), $212(10 \%)$ in the wide-target group ( 5 facilities) and $172(8.4 \%)$ in the high-target group ( 4 facilities). The minimum-maximum target ranges of phosphorus among these non-adherence groups, as reported by each facility's director, were $2.0-6.0 \mathrm{mg} / \mathrm{dL}$ in the low-target group, $2.0-6.5 \mathrm{mg} /$ $\mathrm{dL}$ in the wide-target group and $3.5-7.0 \mathrm{mg} / \mathrm{dL}$ in the high-target group. Most facilities (44/57 facilities) did not change their target ranges from baseline to final measurement. 


\section{Patient characteristics}

Patient baseline characteristics are summarised in table 1. Considering all patients, the mean age was 65.5 years $(\mathrm{SD}=12.4)$, and $65 \%$ of patients were men. The hightarget group had a mean age 2 years lower than that of the other groups. The serum albumin value in the hightarget group was higher $(3.9 \mathrm{~g} / \mathrm{dL})$ than that in the other three groups (average $3.7 \mathrm{~g} / \mathrm{dL}$ ). Regarding patient-level serum phosphorus, the adherence, low-target, and widetarget groups were in the range of serum phosphorus recommended by the CPG for CKD-MBD for approximately $60 \%-70 \%$ of patients. In contrast, less than $50 \%$ of the high-target group patients had phosphorus levels in accordance with the recommendation. The change of serum phosphorus by the groups during the study was presented in online supplemental figure 1 . Over $70 \%$ of patients in the adherence group had serum calcium in the range $8.4-10.0 \mathrm{mg} / \mathrm{dL}$, while the other groups had approximately $50 \%-60 \%$ of patients in that range. The group with the higher UL target also had higher levels of intact PTH. In all groups, most patients had a treatment time of 211-240 min. The high-target group had more patients with less than $211 \mathrm{~min}$ and more than $240 \mathrm{~min}$ compared with the other three groups. Regarding comorbidities, heart disease in the wide-target and high-target groups was proportionally less common than in the other groups. The high-target group was less likely to use prescription drugs related to $\mathrm{CKD}-\mathrm{MBD}$ than were the other three groups.

\section{Facility-level target ranges of phosphorus and patient outcomes}

In terms of all-cause mortality, 329 (16\%) patients died during the observation period. The median follow-up period was 2.97 years (IQR: 1.33-3.00). The mortality rate among all patients was 7.3 per 100 person-years. The mortality rate of the adherence group (6.9 per 100 person-years) was lower than that of the other three categories: 7.7 in the low-target group, 7.5 in the wide-target group and 7.5 in the high-target group per 100 personyears (table 1). The results of the Kaplan-Meier method and the log-rank test were presented in online supplemental materials (online supplemental figures 2 and 3).

With reference to the adherence group, the adjusted HRs $(95 \%$ CIs) for all-cause mortality were 1.04 (0.76 to $1.43 ; \mathrm{p}=0.79)$ for the low-target group and 1.11 (0.68 to $1.81 ; \mathrm{p}=0.68)$ for the wide-target group. The high-target group was significantly associated with greater mortality (adjusted HR=1.95, 95\% CI 1.12 to 3.38, $\mathrm{p}=0.018$; figure 3). Plots of Schoenfeld residuals were consistent with proportionally. In terms of MACEs, this result was consistent with the result of mortality. The detailed results of the analyses were in online supplemental materials (online supplemental tables 1 and 2).

\section{Facility characteristics}

Of the 57 eligible facilities, 27 (47\%) facilities set the institutional target according to the CPG for CKD-MBD, while 30 facilities did not (table 2). Of 24 large facilities (>150 HD patients), most adhered to the CPG (48\%; $13 / 27$ facilities). In contrast, the number of small facilities $(<100 \mathrm{HD}$ patients) in the adherence group (19\%) was less than half compared with the non-adherence group $(40 \%)$. Consultation hours per week were higher in the adherence facilities than in the non-adherence facilities; $67 \%$ of facilities that adhered to the CPG provided over 60 hours of consultation, while this percentage was $33 \%$ among facilities that did not adhere to the CPG. Regarding healthcare professionals caring for HD patients, a greater percentage of facilities with dieticians and certified social workers belonged to the adherence group than the nonadherence group (81\% vs $50 \%$, and $52 \%$ vs $33 \%$, respectively); the pattern reversed for nurses certified in dialysis nursing ( $0 \%$ vs $13 \%)$.

\section{Facility factors associated with facility-level guideline adherence}

In univariate logistic regression analyses, four factors were selected as candidates for the final model: facility size, consultation hours, presence of dietician, and presence of certified social worker. In the multivariate logistic regression analysis, the association between dietician presence and facility-level guideline adherence was statistically significant (adjusted OR 4.51, 95\% CI 1.15 to 17.7, $\mathrm{p}=0.031$; table 3$)$. The c-statistic $(95 \% \mathrm{CI})$ of the final model was 0.77 (0.65 to 0.89$)$.

\section{DISCUSSION}

This study evaluated whether facility-level adherence to guidelines for the target range of phosphorus was associated with mortality in Japanese dialysis patients, and investigated facility-related factors associated with guideline adherence. Approximately $47 \%$ of facilities adopted treatment policies consistent with the CPG for phosphorus management. Our results showed a significant association between facility-level guideline adherence regarding target ranges of serum phosphorus and patient mortality; facilities with higher target ranges demonstrated higher mortality rates. Moreover, involving dieticians in the treatment of dialysis patients was associated with facility-level guideline adherence to target ranges for phosphorus.

Our findings help to understand the effects of facilitylevel guideline adherence on patient mortality. Overall, patients' actual serum phosphorus values showed trends similar to the corresponding facility target ranges. Compared with the adherence group, the low-target group exhibited a large proportion of cases with serum phosphorus below $3.5 \mathrm{mg} / \mathrm{dL}$, the wide-target group had a higher percentage of cases with values $<3.5 \mathrm{mg} / \mathrm{dL}$ or $>6.0 \mathrm{mg} / \mathrm{dL}$, and approximately half of the patients in the high-target group demonstrated values $>6.0 \mathrm{mg} / \mathrm{dL}$. Previous studies showed an association between patientlevel serum phosphorus measured at baseline and allcause mortality. ${ }^{12} 13$ Patients' serum phosphorus levels below $3.5 \mathrm{mg} / \mathrm{dL}$ and above $6.5 \mathrm{mg} / \mathrm{dL}$ were significantly 


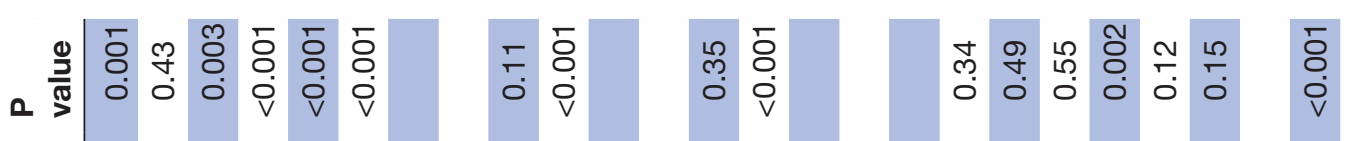

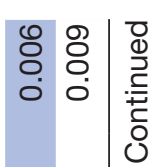

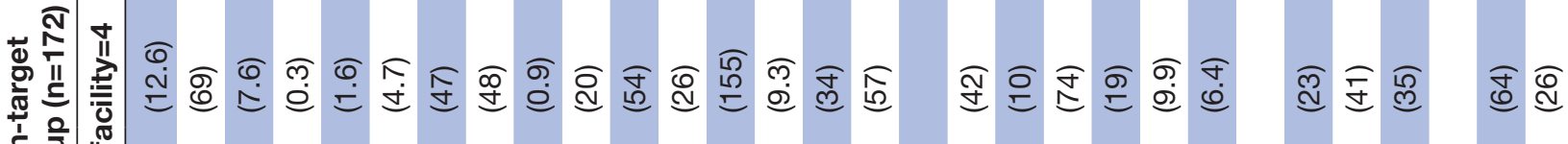

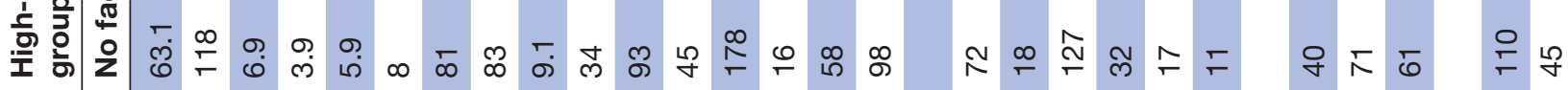

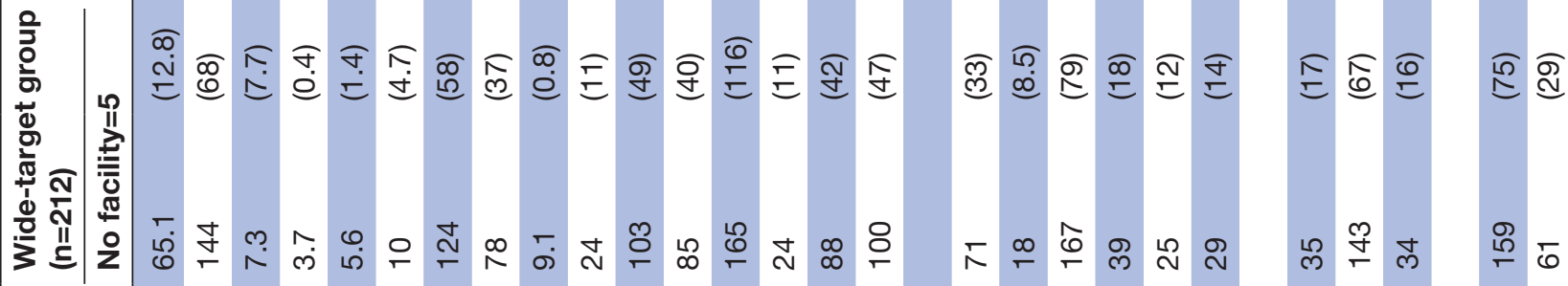

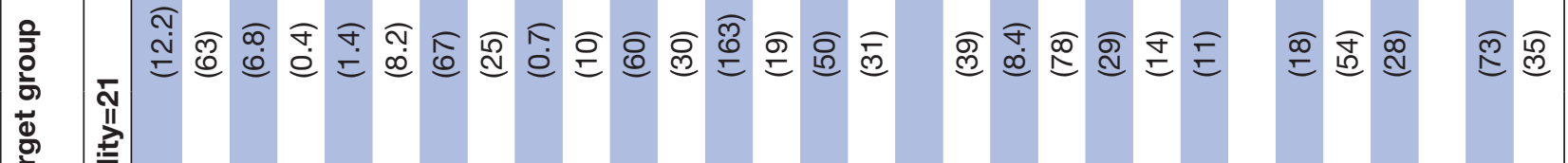

के 2 के

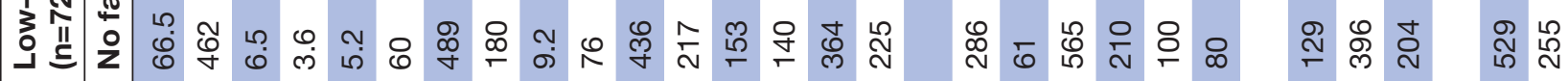

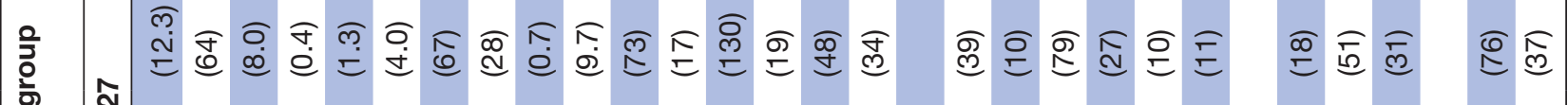

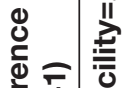

至恶

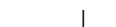

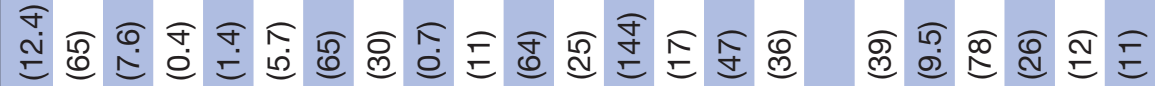

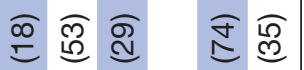

เి 疍

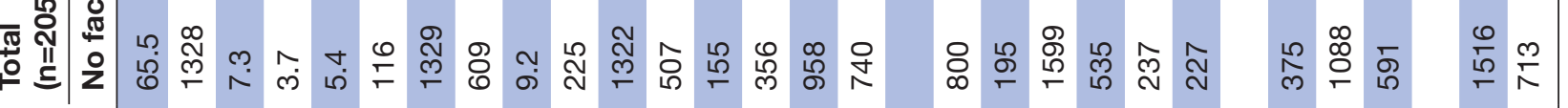



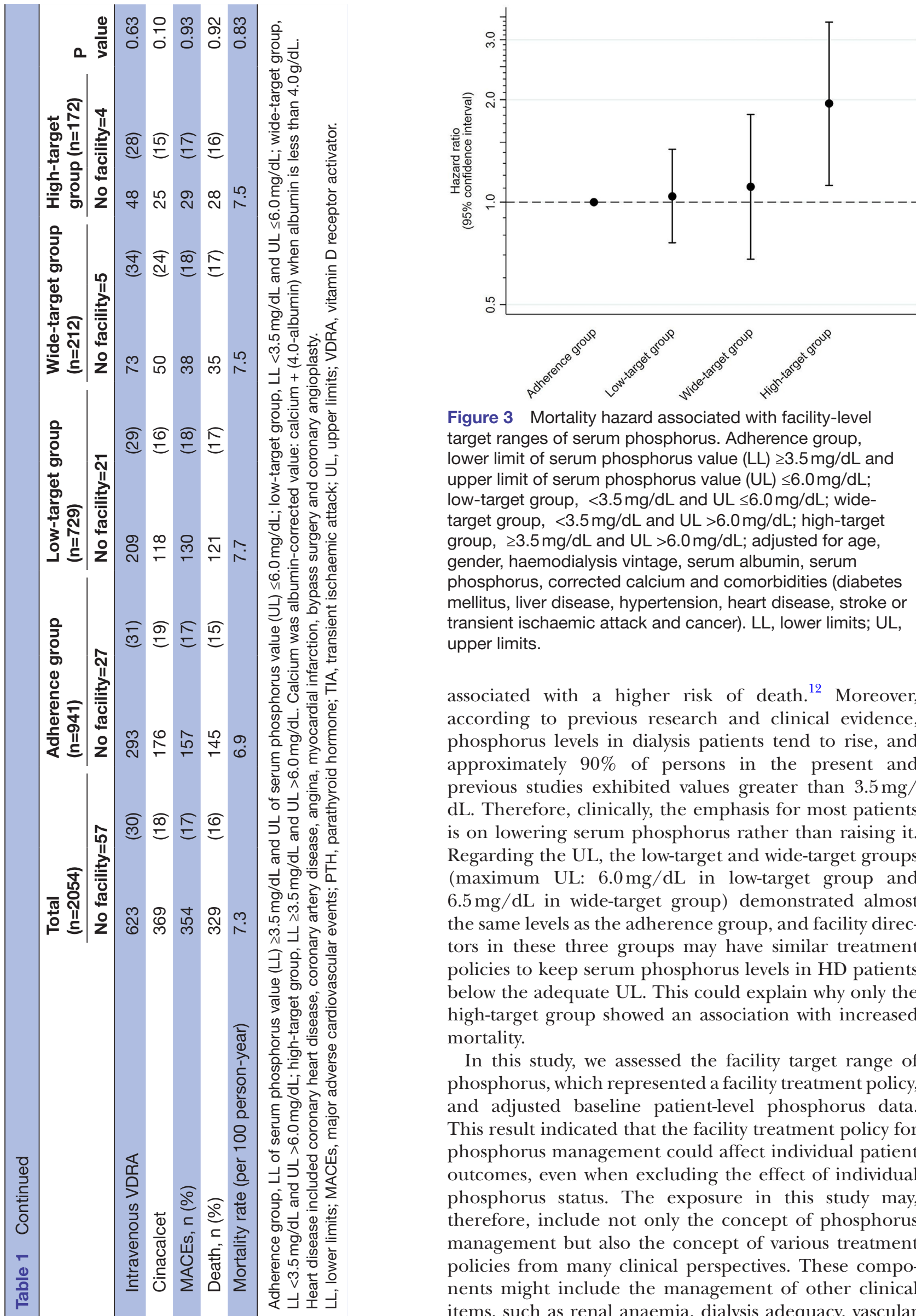

Figure 3 Mortality hazard associated with facility-level target ranges of serum phosphorus. Adherence group, lower limit of serum phosphorus value (LL) $\geq 3.5 \mathrm{mg} / \mathrm{dL}$ and upper limit of serum phosphorus value (UL) $\leq 6.0 \mathrm{mg} / \mathrm{dL}$; low-target group, $<3.5 \mathrm{mg} / \mathrm{dL}$ and $\mathrm{UL} \leq 6.0 \mathrm{mg} / \mathrm{dL}$; widetarget group, $<3.5 \mathrm{mg} / \mathrm{dL}$ and $\mathrm{UL}>6.0 \mathrm{mg} / \mathrm{dL}$; high-target group, $\geq 3.5 \mathrm{mg} / \mathrm{dL}$ and UL $>6.0 \mathrm{mg} / \mathrm{dL}$; adjusted for age, gender, haemodialysis vintage, serum albumin, serum phosphorus, corrected calcium and comorbidities (diabetes mellitus, liver disease, hypertension, heart disease, stroke or transient ischaemic attack and cancer). LL, lower limits; UL, upper limits.

associated with a higher risk of death. ${ }^{12}$ Moreover, according to previous research and clinical evidence, phosphorus levels in dialysis patients tend to rise, and approximately $90 \%$ of persons in the present and previous studies exhibited values greater than $3.5 \mathrm{mg}$ / dL. Therefore, clinically, the emphasis for most patients is on lowering serum phosphorus rather than raising it. Regarding the UL, the low-target and wide-target groups (maximum UL: $6.0 \mathrm{mg} / \mathrm{dL}$ in low-target group and $6.5 \mathrm{mg} / \mathrm{dL}$ in wide-target group) demonstrated almost the same levels as the adherence group, and facility directors in these three groups may have similar treatment policies to keep serum phosphorus levels in HD patients below the adequate UL. This could explain why only the high-target group showed an association with increased mortality.

In this study, we assessed the facility target range of phosphorus, which represented a facility treatment policy, and adjusted baseline patient-level phosphorus data. This result indicated that the facility treatment policy for phosphorus management could affect individual patient outcomes, even when excluding the effect of individual phosphorus status. The exposure in this study may, therefore, include not only the concept of phosphorus management but also the concept of various treatment policies from many clinical perspectives. These components might include the management of other clinical items, such as renal anaemia, dialysis adequacy, vascular 


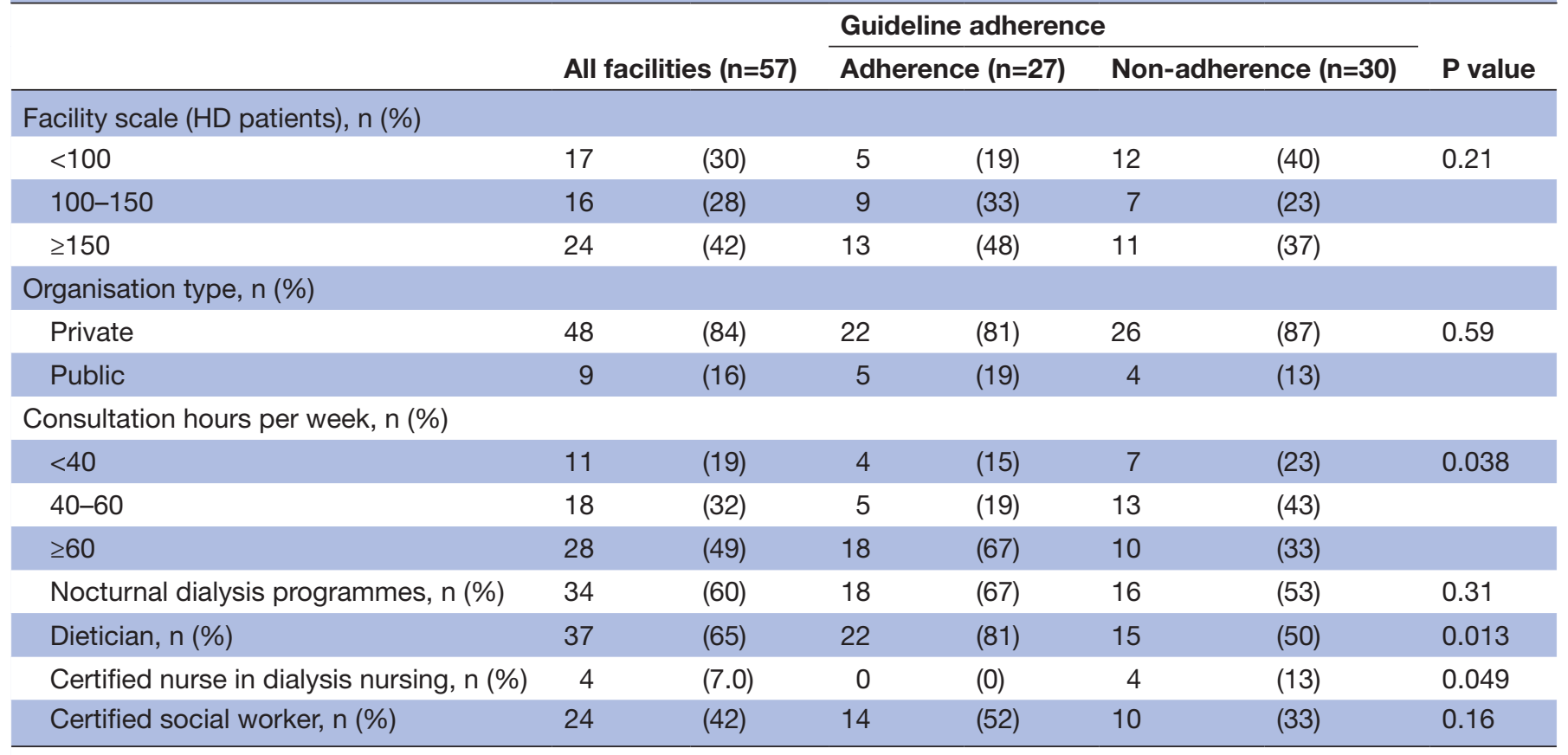

Dietician, certified nurse in dialysis nursing and certified social worker were defined as whether the facility had at least one such person in charge of HD patients.

$\mathrm{HD}$, haemodialysis.

access and comorbidity, as well as patient education. Facilities that set the same target ranges of phosphorus as those indicated in the CPG may tend to offer highquality care to patients. Therefore, even if individual-level effects at baseline were detected, there was an association between facility treatment policy and mortality. That is, this association between exposure and mortality in our study may indicate the composite effects of institutional treatment strategies.

There could be several reasons why dialysis facilities did not set their targets according to the CPG. First, some physicians were sceptical about guidelines and related evidence; they thought, for example, that the guidelines were too rigid to apply to individual patients, and not appropriate for translation to typical patients with different demographic characteristics. ${ }^{15-1729}$ Generally, the practice of evidence-based medicine is described as "the integration of best research evidence with clinical expertise and patient values. ${ }^{30}$ This does not mean that medical staff blindly provide uniform treatment to all patients based on CPGs. A previous systematic review reported the two main reasons for intentional non-adherence to guidelines were contraindications and patients' preferences. ${ }^{31}$ The main strategies for phosphate control are diet restrictions and dose adjustment of phosphate binders, which could harm some patients. For example, strict diet restrictions could worsen nutritional status, while increasing phosphate binders may lead to polypharmacy or constipation. Physicians who consider these potential harms could accept a less strict phosphorus target. Our findings that patients in the high-target group were less likely to take phosphate binders support this idea. Moreover, CPGs may not be fully known to physicians although the CPG for CKDMBD is available on an open-access website.

Additionally, this study indicated that facilities where the treatment policy was consistent with the CPG recommendation were more likely to involve dieticians in patients' treatment. Dieticians specialise not only in phosphorus management but also in many other aspects of nutrition management, including renal-disease-related intake restriction of salt, potassium and water, and comorbidities such as diabetes mellitus and hyperlipidaemia. The facilities where dieticians are involved might offer multifaceted patient education. Further research is needed in other clinical fields, since it is possible that facility-level guideline adherence may be associated with the presence of relevant healthcare professionals.

\section{Strengths and limitations}

The first strength of the current study was its design. ${ }^{32}$ The measurement of exposure had high validity, as we collected the exposure data prospectively to prevent recall bias. The second advantage was the sample size. This study included a relatively large population and numerous dialysis centres. Therefore, we were able to evaluate facility-level factors. The third strength was the sampling method of the J-DOPPS, which was representative of most Japanese dialysis settings. ${ }^{32}$

There were several limitations to our study. First, facilitylevel target ranges of phosphorus at baseline were used to define the exposure categories in this study; however, patients may have transferred into different categories 
Table 3 Facility factors associated with facility-level guideline adherence

\begin{tabular}{|c|c|c|c|c|c|c|}
\hline & \multicolumn{3}{|c|}{ Univariate model } & \multicolumn{3}{|c|}{ Multivariate model } \\
\hline & OR & $95 \% \mathrm{Cl}$ & $P$ value & OR & $95 \% \mathrm{Cl}$ & $P$ value \\
\hline \multicolumn{7}{|c|}{ Facility scale (HD patients) } \\
\hline $100-150$ & 3.09 & 0.73 to 13.0 & 0.12 & 1.48 & 0.29 to 7.62 & 0.64 \\
\hline$\geq 150$ & 2.84 & 0.76 to 10.6 & 0.12 & 1.02 & 0.19 to 5.46 & 0.98 \\
\hline Private & Reference & & & & & \\
\hline Public & 1.48 & 0.35 to 6.19 & 0.59 & & & \\
\hline \multicolumn{7}{|c|}{ Consultation hours per week } \\
\hline$<40$ & Reference & & & Reference & & \\
\hline $40-60$ & 0.67 & 0.14 to 3.35 & 0.63 & 0.55 & 0.08 to 3.62 & 0.54 \\
\hline Offered & 1.75 & 0.60 to 5.13 & 0.31 & & & \\
\hline \multicolumn{7}{|l|}{ Dietician } \\
\hline Absent & Reference & & & Reference & & \\
\hline Present & 4.40 & 1.32 to 14.7 & 0.016 & 4.51 & 1.15 to 17.7 & 0.031 \\
\hline \multicolumn{7}{|c|}{ Certified social worker } \\
\hline Absent & Reference & & & Reference & & \\
\hline Present & 2.15 & 0.74 to 6.28 & 0.16 & 1.67 & 0.48 to 5.82 & 0.42 \\
\hline
\end{tabular}

Dietician and certified social worker were defined as whether the facility had at least one such person in charge of HD patients. C-statistic of the final model was $0.77(95 \% \mathrm{Cl} 0.65$ to 0.89$)$.

$\mathrm{HD}$, haemodialysis.

during the study period. Further, as the facility treatment policy and patient data were collected at different times, misclassification might have also occurred. However, we confirmed that the facility target ranges changed minimally, and that measurement times differed only slightly throughout the observation period. Second, the facility treatment policy might not be an aggregate opinion in each facility. This is because the facility-level target range of phosphorus was reported by one facility director; if there were multiple doctors in a centre, there might not have necessarily been consensus on the target range. Third, our findings did not imply that setting facility treatment targets according to CPGs will directly reduce mortality. Fourth, the adjustment of unknown confounding factors associated with the relationships we investigated was not possible, which is a general limitation of observational studies. Finally, it may be difficult to generalise our findings to other areas or guidelines, as the present study did not investigate other types of CPGs or countries.

\section{CONCLUSION}

Our findings demonstrated that approximately half of the dialysis centres in Japan set target ranges according to the CPG for phosphorus management. We found an association between a higher target range of serum phosphorus and patient mortality. Our results also suggested that the presence of a healthcare professional involved with the facility-level treatment policy was associated with facility-level guideline adherence. These results suggest that facility directors and healthcare providers should refer to updated CPGs when evaluating treatment policies in their facilities. Furthermore, physicians and other healthcare professionals who are relevant to facility treatment policies may play a key role in guideline adherence.

Author affiliations

${ }^{1}$ Department of Healthcare Epidemiology, Graduate School of Medicine and Public Health, Kyoto University, Kyoto, Japan

${ }^{2}$ Section of Clinical Epidemiology, Department of Community Medicine, Graduate School of Medicine, Kyoto University, Kyoto, Japan

${ }^{3}$ Institute for Health Outcomes and Process Evaluation Research (iHope International), Kyoto, Japan

${ }^{4}$ Department of Emergency Medicine, Kobe City Medical Center General Hospital, Kobe, Japan

${ }^{5}$ Center for Innovative Research for Communities and Clinical Excellence (CiRCLE), Fukushima Medical University, Fukushima, Japan

${ }^{6}$ Shirakawa STAR for General Medicine, Fukushima Medical University, Fukushima, Japan

Acknowledgements We would like to thank the Arbor Research Collaborative for Health (Ann Arbor, MI, USA) for administering the J-DOPPS, and express our 
appreciation for the support provided by Kyowa Kirin, for allowing the use of data without restrictions on publication. The DOPPS.org website lists the full details. We also thank the study nurses, physicians and medical directors for all the time and attention they devoted to our study.

Contributors TI conceived and designed the study. SS, SF, and YY supervised the conduct of the study. TI collected and analysed the data and drafted the manuscript. SS, TH, YM, SF, and YY reviewed and made substantial contributions to earlier drafts. All authors have read and approved the final version of the manuscript.

Funding This study was funded by Kyowa Kirin (grant number N/A) and the Japan Society for the Promotion of Science (JSPS) KAKENHI (grant number 19J23232).

Competing interests SF has acted as a scientific advisor to Kyowa Kirin. TI, SS, $\mathrm{TH}, \mathrm{YM}$ and $\mathrm{YY}$ have no relevant financial interests.

Patient consent for publication Not required.

Ethics approval All participants in the study provided written informed consent prior to study enrolment. This study was approved by Kyoto University Graduate School and Faculty of Medicine Kyoto University Hospital Ethics Committee (approval number R1301).

Provenance and peer review Not commissioned; externally peer reviewed.

Data availability statement № data are available. The data that support the findings of this study are available from the Arbor Research Collaborative for Health, but restrictions apply to the availability of these data, which were used under license for the current study, and so are not publicly available. However, data requests can be sent to Arbor Research via their website (http://www. arborresearch.org/AboutUs/ContactUs.aspx).

Supplemental material This content has been supplied by the author(s). It has not been vetted by BMJ Publishing Group Limited (BMJ) and may not have been peer-reviewed. Any opinions or recommendations discussed are solely those of the author(s) and are not endorsed by BMJ. BMJ disclaims all liability and responsibility arising from any reliance placed on the content. Where the content includes any translated material, BMJ does not warrant the accuracy and reliability of the translations (including but not limited to local regulations, clinical guidelines, terminology, drug names and drug dosages), and is not responsible for any error and/or omissions arising from translation and adaptation or otherwise.

Open access This is an open access article distributed in accordance with the Creative Commons Attribution Non Commercial (CC BY-NC 4.0) license, which permits others to distribute, remix, adapt, build upon this work non-commercially, and license their derivative works on different terms, provided the original work is properly cited, appropriate credit is given, any changes made indicated, and the use is non-commercial. See: http://creativecommons.org/licenses/by-nc/4.0/.

\section{ORCID iDs}

Takahiro Itaya http://orcid.org/0000-0002-0560-8686

Sayaka Shimizu http://orcid.org/0000-0001-9283-144X

Yosuke Yamamoto http://orcid.org/0000-0003-1104-2612

\section{REFERENCES}

1 Worrall G, Chaulk P, Freake D. The effects of clinical practice guidelines on patient outcomes in primary care: a systematic review. CMAJ 1997;156:1705-12.

2 Andrews EJ, Redmond HP. A review of clinical guidelines. Br J Surg 2004;91:956-64.

3 Brown PD. Adherence to guidelines for community-acquired pneumonia: does it decrease cost of care? Pharmacoeconomics 2004;22:413-20.

4 Bansilal S, Castellano JM, Garrido E, et al. Assessing the Impact of Medication Adherence on Long-Term Cardiovascular Outcomes. $J$ Am Coll Cardiol 2016;68:789-801.

5 Hanney WJ, Masaracchio M, Liu X, et al. The influence of physical therapy guideline adherence on healthcare utilization and costs among patients with low back pain: a systematic review of the literature. PLoS One 2016;11:e0156799.

6 Parikh K, Hall M, Blaschke AJ, et al. Aggregate and hospital-level impact of national guidelines on diagnostic resource utilization for children with pneumonia at children's hospitals. J Hosp Med 2016;11:317-23.

7 Grol R, Dalhuijsen J, Thomas S, et al. Attributes of clinical guidelines that influence use of guidelines in general practice: observational study. BMJ 1998;317:858-61.
8 McGlynn EA, Asch SM, Adams J, et al. The quality of health care delivered to adults in the United States. N Engl J Med 2003;348:2635-45.

9 Cabana MD, Rand CS, Powe NR, et al. Why don't physicians follow clinical practice guidelines? A framework for improvement. JAMA 1999;282:1458-65.

10 Kidney Disease: Improving Global Outcomes (KDIGO) CKD-MBD Update Work Group. KDIGO 2017 Clinical Practice Guideline Update for the Diagnosis, Evaluation, Prevention, and Treatment of Chronic Kidney Disease-Mineral and Bone Disorder (CKD-MBD). Kidney Int Suppl 2017;7:1-59.

11 Tattersall J, Martin-Malo A, Pedrini L, et al. EBPG guideline on dialysis strategies. Nephrol Dial Transplant 2007;22 Suppl 2:ii5-21.

12 Young EW, Albert JM, Satayathum S, et al. Predictors and consequences of altered mineral metabolism: the dialysis outcomes and practice patterns study. Kidney Int 2005;67:1179-87.

13 Palmer SC, Hayen A, Macaskill P, et al. Serum levels of phosphorus, parathyroid hormone, and calcium and risks of death and cardiovascular disease in individuals with chronic kidney disease: a systematic review and meta-analysis. JAMA 2011;305:1119-27.

14 Grol R. Implementing guidelines in general practice care. Qual Health Care 1992;1:184-91.

15 Carlsen B, Bringedal B. Attitudes to clinical guidelines--do GPs differ from other medical doctors? BMJ Qual Saf 2011;20:158-62.

16 Lugtenberg M, Burgers JS, Besters CF, et al. Perceived barriers to guideline adherence: a survey among general practitioners. $B M C$ Fam Pract 2011;12:98.

17 Farquhar CM, Kofa EW, Slutsky JR. Clinicians' attitudes to clinical practice guidelines: a systematic review. Med J Aust 2002;177:502-6.

18 Lugtenberg M, Burgers JS, Westert GP. Effects of evidence-based clinical practice guidelines on quality of care: a systematic review. Qual Saf Health Care 2009;18:385-92.

19 Okelo SO, Butz AM, Sharma R, et al. Interventions to modify health care provider adherence to asthma guidelines: a systematic review. Pediatrics 2013;132:517-34.

20 Bighelli I, Ostuzzi G, Girlanda F, et al. Implementation of treatment guidelines for specialist mental health care. Cochrane Database Syst Rev 2016;12:CD009780.

21 Prior M, Guerin M, Grimmer-Somers K. The effectiveness of clinical guideline implementation strategies--a synthesis of systematic review findings. J Eval Clin Pract 2008;14:888-97.

22 Young EW, Goodkin DA, Mapes DL, et al. The dialysis outcomes and practice patterns study (DOPPS): an international hemodialysis study. Kidney Int 2000;57:S74-81.

23 Pisoni RL, Gillespie BW, Dickinson DM, et al. The dialysis outcomes and practice patterns study (DOPPS): design, data elements, and methodology. Am J Kidney Dis 2004;44:7-15.

24 Fukagawa M, Yokoyama K, Koiwa F, et al. Clinical practice guideline for the management of chronic kidney disease-mineral and bone disorder. Ther Apher Dial 2013;17:247-88.

25 The Japanese Society for Dialysis Therapy. Clinical practice guideline for the management of chronic kidney disease-mineral and bone disorder. J Jpn Soc Dial Ther 2012;45:301-56.

26 Wang C, Kane R, Levenson M, et al. Association between changes in CMS reimbursement policy and drug labels for ErythrocyteStimulating agents with outcomes for older patients undergoing hemodialysis covered by fee-for-service Medicare. JAMA Intern Med 2016;176:1818-25

27 Saran R, Bragg-Gresham JL, Levin NW, et al. Longer treatment time and slower ultrafiltration in hemodialysis: associations with reduced mortality in the DOPPS. Kidney Int 2006;69:1222-8.

28 Payne RB, Little AJ, Williams RB, et al. Interpretation of serum calcium in patients with abnormal serum proteins. Br Med $J$ 1973;4:643-6.

29 Carlsen B, Glenton C, Pope C. Thou shalt versus thou shalt not: a meta-synthesis of GPS' attitudes to clinical practice guidelines. $\mathrm{Br} \mathrm{J}$ Gen Pract 2007:57:971-8.

30 Sackett DL, Straus SE, Richardson WS. Evidence-Based medicine: how to practice and teach EBM. 2nd ed. London, UK: Churchill Livingstone, 2000.

31 Arts DL, Voncken AG, Medlock S, et al. Reasons for intentional guideline non-adherence: a systematic review. Int J Med Inform 2016;89:55-62.

32 Robinson BM, Bieber B, Pisoni RL, et al. Dialysis outcomes and practice patterns study (DOPPS): its strengths, limitations, and role in informing practices and policies. Clin J Am Soc Nephrol 2012;7:1897-905. 